\title{
A mobile system to increase efficiency of the lecturers when preventing academic dishonesty during written exams
}

\author{
Pedro Maroco, João Cambeiro, Vasco Amaral \\ Departamento de Informática \\ Faculdade de Ciências e Tecnologia \\ Universidade Nova de Lisboa \\ Lisboa, Portugal \\ pmaroco,jmc12976@campus.fct.unl.pt and vma@fct.unl.pt
}

\begin{abstract}
The process of in-class control and monitoring of students' examination activities is traditionally conducted manually, without the support of technology. The workflow can include tasks for attendance record or dishonesty control. The tasks include identifying the students before, during and after the beginning of the evaluation activity. Can also include examination room allocation to the activity, as well as the distribution of students per available examination rooms and distribution of students inside the room according to a classroom seating plan (which can have a varied setup).

However, with a large number of students, the execution of these tasks becomes challenging to perform efficiently in a manual fashion, requiring innovative techniques or tools to support them effectively.

In this work, we propose the design and implementation of a mobile system based on IoT technology and MIFARE student's cards to support the lecturer efficiently during the examination workflow. We propose the use of current indoor location technology, and we build a proof of concept prototype, validating it in a real educational environment.
\end{abstract}

Index Terms-Internet-of-things, Education Technology, Indoor Location

\section{INTRODUCTION}

Especially in the case of the education system, there are management tasks such as schedule distribution or back office processes using IT solutions to assist and help lecturers to carry them out [1], [2]. However, plenty of tasks are still not supported by proper technological solutions and could be improved by developing a specific solution to support their achievement. For example, managing logistics inside and outside an exam room during the evaluation activity can be a difficult task to perform. Such kind of tasks sometimes involves a significant number of students (in the order of magnitude of hundreds), making it challenging to have efficient management in such environments. Although existing hardwired presence control systems for buildings, it is a costly solution that typically is featured for examination. Therefore, a mobile system, adequate for any building and retrofitting environments, could be designed to perform a set of tasks aimed to improve efficiency and productivity during evaluation activities in an exam room environment.
Among other possibilities, the proposed system can help on tasks such as monitoring attendance and authenticity to avoid academic dishonesty, path optimisation during the beginning of the activity and monitoring environmental quality during the evaluation activity such as noise levels or temperature.

Based on the problems identified earlier, we are interested in solving the problem of more efficiently controlling and monitoring the exam room environment during exams time. Our system intends to automate and assist all the activities related to the exam, knowing beforehand that the variability of the rooms' physical layout can be broad. The validation process involves: i) to produce a seating plan; ii) log the activity for later analysis of possible fraud; and, iii) check conformance with the seating plan. Certain aspects, such as different sizes of rooms and their sit places arrangement, lead to varying possibilities of physical students' distribution. This variability requires that the proposed solution must be highly adaptable to different environments.

This paper is organised as follows: Section II describes the process of a typical exam scenario. Section III reports previous related studies on solutions aimed to solve the same problem presented at the beginning of this section. Section IV describes how the prototype was built, describing the conceptualised solution, features and limitations. Section V presents the methodology and the setup used to test the system, followed by an analysis of the performance results obtained. Section VI presents conclusions and future work.

\section{TyPiCAL IN-Class EXAMINATION SCENARIOS}

Nowadays, the control and management of the student's inroom examination procedures are put into practice by the lecturer (also called examiner) without technology support. This situation is especially true in under-developed countries where it is not economically viable to have building infrastructures with hardwired automation since their conception.

In some institutions, the number of students for examination exceeds several hundred. The typical scenario is when the student is informed beforehand about the date, starting times and room(s) where the event will take place. 
Usually, before starting the exam, each student must wait (probably queuing in front of the doorway until being allowed to get inside the room). Sometimes, attendance control must be done at the entrance of the room, with the penalty of being a time-consuming activity, to check whether if the student is authorised for that event.

After entering the room, students can either choose their sit or have a guided procedure. For instance, there can be some sort of distribution criteria like alphabetically, or by student's number, random, or cimply introduced by the lecturer.

The student has to take with him his ID (or student's card) to verify identity. This way, it is possible to check for one type of academic dishonesty, which is the case of another person trying to impersonate the real student. This validation can also be used to verify that the student is correctly enrolled in the examination activity: did proper registration in the event; paid for the adequate fee, if that is the case; and, is even enrolled in the course.

Sometimes, It can also be the case that the lecturer registers the sitting position of each student in the room to log this information for later use. This validation can be used to crosscheck situations of a possible copy in between students in neighbour positions. For each student, their student number (or other id information) and their position within the examination room should be recorded. This can be done before starting, during or after the exam. All situations have drawbacks. The first option is very time consuming and might worsen the stress effect of the students while waiting. The second option might disrupt the student's concentration. The last choice implies that all the students have to synchronise and wait for the stipulated end of the exam. Additionally, the student will have to wait for an extended period of verification. Independently of the moment when the check is done, this is an error-prone, timeconsuming and laborious task.

After the moment of confirmation of the student's ID (that it is the person supposed to be, in the right place, and duly registered), there is the moment of handling with the finished exams. This moment can have several variations in procedure but usually implies that the student hands back the written exam or the lecturer collects it. Also, each student might be asked to show the identification again. Finally, the student can leave the room.

\section{RELATED WORK}

Fraud and cheating during evaluation activities is a wellknown problem and has been studied through different approaches and scenarios [3]. Nowadays, there are several solutions to tackle this type of fraud in online evaluations that have become increasingly popular approaches [4].

However, in the context of in-class examinations, we claim that there is not yet an automated solution to help lecturers to simultaneously manage, control the evaluation process and enforce fraud prevention by using geolocation techniques.

Nonetheless, the solution presented in this paper does not dismiss visual control, which allows the detection of infringements, such as the use of cheating papers.

\section{Prototype Implementation}

This section intends to present the application's use case and the prototype of our proposed mobile examination identification control system.

\section{A. Architecture}

Regarding the system conceptualised in Section I, the architecture of the developed system is represented in Figure 1.

\section{B. E-Presence App}

The flowchart of Figure 2 accounts for the application workflow, followed by a detailed description of the several features developed.

1) Login: At the first use of the application, the lecturer is presented with the authentication fields to $\log$ in to the application. The lecturer must use his/her academic credentials which, depending on their status, will have access to the various functionalities of the application.

To avoid successive user authentication during an evaluation activity, the session should remain active for a certain period. If the user signs out or the session time expires, authentication will be required again.

2) Courses: In this section, the lecturer can select the course he wants to manage from a list of courses which he is responsible for. Depending on the option selected, classes, students enrolled and evaluation activities regarding the selected course will be loaded in the application.

3) Classes: This section allows the user to check the class schedule and monitor student attendance at classes. Upon entering this section, the list of turns for which the user is responsible is displayed. The user can check the list of classes of a certain turn by clicking on it.

After selecting the desired class, it is possible to check the attendance list or start the attendance control using a connected card reader or by manual mode. Also, the user can export the list of participants at any time.

4) Students: This section, depicted in Figure 3, allows the user to check the list of students enrolled in the selected course. In addition to the name and student number, it is possible to check if a student already has his/her student card registered in the application. This information is presented through a coloured label next to each element of the list. It is possible to check the details of a particular student such as the summary of their attendances by selecting the desired element from the student list.

If a student does not have a registered student card or the card has been replaced, a new association can be made using the card reader.

5) Evaluations: The evaluations section allows the user to check the evaluation activity schedule and monitor student attendances during evaluation activities. In the first screen that appears, the user can check the list of planned evaluation activities for which the user is responsible or schedule a new evaluation activity.

Details such as the device IP address, the list of students participating in a specific evaluation activity or the validation 


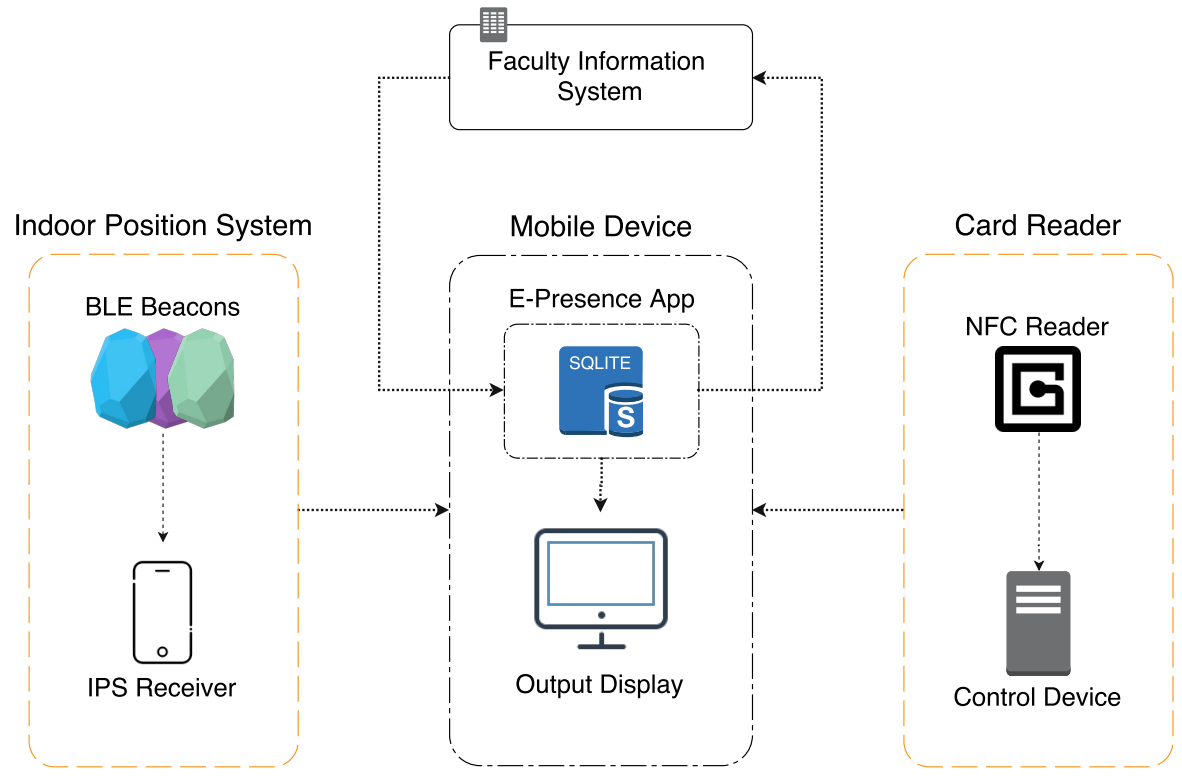

Fig. 1. System architecture diagram

status of each student can be checked if an evaluation activity is selected.

Also, it is possible to connect a card reader at any time. If no sensor is connected or if the student card cannot be used, the validation must be done manually by the student number.

Whenever a student card is detected for a new validation, the system will check which was the last validation executed for the same card number to decide which will be the next validation that should be executed. If the student is enrolled for the evaluation activity and their presence has not yet been registered, the entrance validation is performed. During this validation, the system will assign a random free seat for the student to do the evaluation activity. If there are no more open seats, an error message will be displayed, and the student's attendance will not be registered.

If the student's presence has already been recorded, the inplace validation is performed. During this phase, the system begins by loading the student's data, such as the name, student number and assigned seat position to be displayed during visual confirmation by the faculty member. If the visual confirmation of the face of the student is accepted, the student's geographical position validation will begin. To perform this task, the application receives the position data from the IPS and verifies if the student is within a radius threshold established from the seat location assigned. If the received position is outside the radius or if the application is unable to communicate with the IPS, a message will be displayed with a proper error message.

Finally, if the student's in-place validation was performed successfully, the system will carry out the delivery validation phase. In this stage, the system will display the student's data, such as photo, name and student number, followed by
Delivered or Not Delivered options that should be selected appropriately regarding the delivery of the evaluation's answer sheet.

\section{Indoor Positioning System}

Due to certain limitations detailed in subsection V-B, an iOS application was developed to send the student location at the time of in-place validation to the application (Fig. 4).

To send the position coordinates, a service was created in the Android application that creates an HTTP server to receive the IPS data. The connection between the IPS and the app is established through a TCP socket, using the IP address and the server port of the device running the application. The messages exchanged by the IPS and the app are encrypted, and their integrity is verified to prevent message tampering.

Each time the IPS updates the coordinate values, a new message is sent containing a JSON object with the updated position value. If the IPS service is disabled, the communication socket is closed.

\section{Card Reader}

The card reader (Fig. 5) communicates with the application through a secure wireless AP that is set by the reader as soon as it starts operating. To pair the reader in the application, the user must enter the specific sensor number which, through the implemented logic, obtain the authentication credentials of the reader in the database.

Whenever a card is detected, the Unique ID (UID) from the MIFARE chip is read and sent to the application.

\section{VALIDATION}

To define the main goal of this section, the template formulated in [5] has been followed: Analyze the behaviour of the 


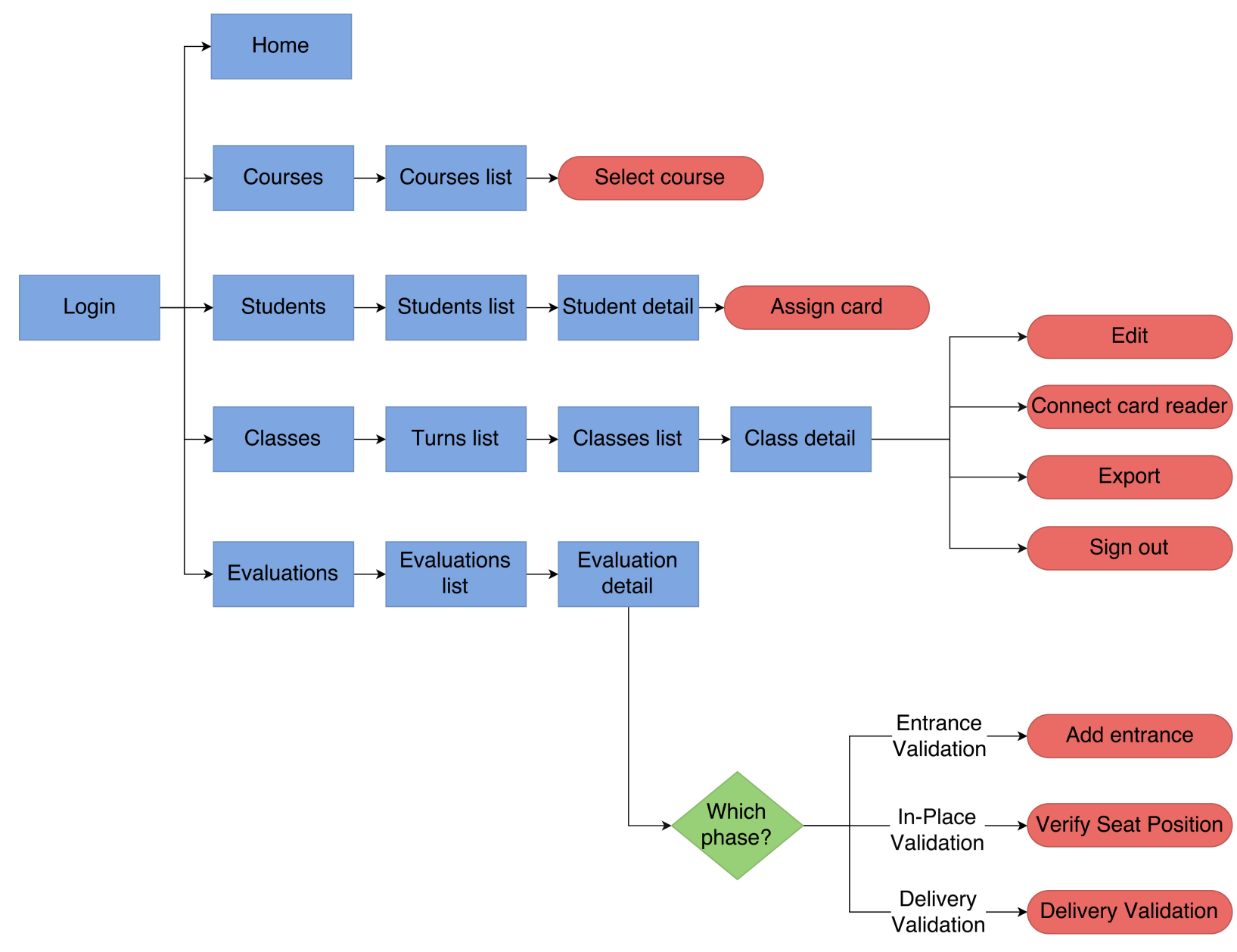

Fig. 2. E-Presence app flowchart

system developed for the purpose of evaluating with respect to the time performance of the system, from the point of view of researchers in the context of an experiment conducted using a real case scenario with participants from [removed for blind review purpose]our academic institution.

\section{A. Case Study Environment}

The classroom used to perform the system evaluation was fitted with 8 BLE beacons from Estimote, covering 39,85 $\mathrm{m}^{2}$ of room area with an accuracy of $+/-1$ meter [6]. Each valid seat was identified with a label containing a row letter and a seat number. After the layout of the sitting positions (or tables) has been set, the coordinates of each valid seat were measured with the purpose of creating a simplified Building Information Model (BIM) of the room, using JSON notation to be loaded within the Android app. Figure 6 shows the room setup used to conduct the evaluation activity.

\section{B. Prototype Setup}

The hardware used during the evaluation process was the following:
- MIFARE Student Cards - Containing MIFARE Classic chips; [7]

- Raspberry Pi 2 - Model B+ with NFC Reader - Reader Identiv SCL3711 supporting the ISO/IEC 14443 Type A and NFC protocol; [8]

- iOS Device - Iphone 6 running iOS v9.2.1 (13D15);

- Android Device - OnePlus One running Android v6.0.1;

- 8 Estimote Proximity Beacons - Hardware v3.3 running firmware A3.2.0. [9]

The use of different devices was because, at the moment of prototype development, the Estimote Indoor SDK supported only iOS platforms. Also, the Android device used was not compatible with the MIFARE contactless IC available in the student cards.

However, this solution can be centralised in future versions, discarding the Raspberry card reader. Supporting both Android and iOS platforms will be possible. Since the prototype development, the Estimote Indoor SDK was made available for the Android platform, and by using an Android device with an NXP NFC reader compatible with MIFARE ICs and 


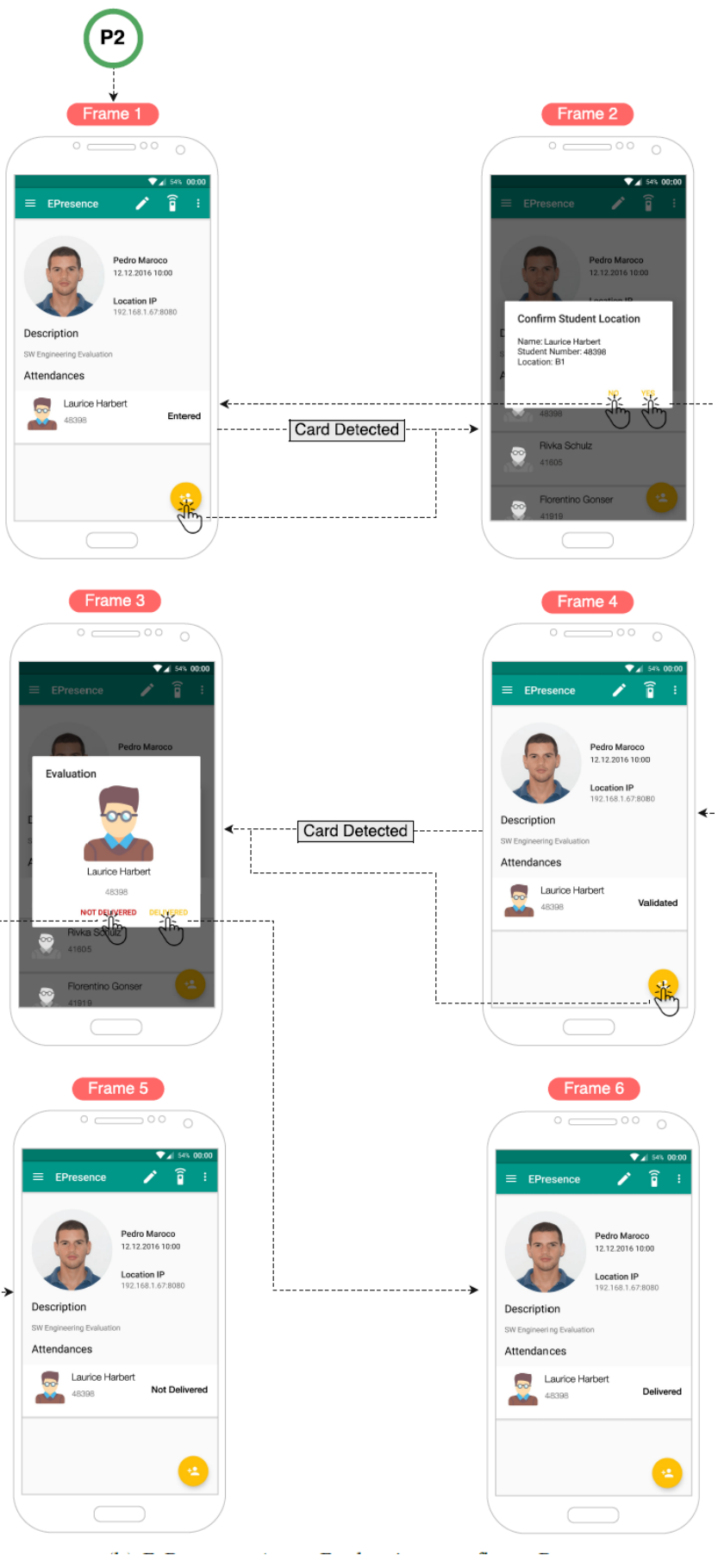

Fig. 3. E-Presence examination control use case

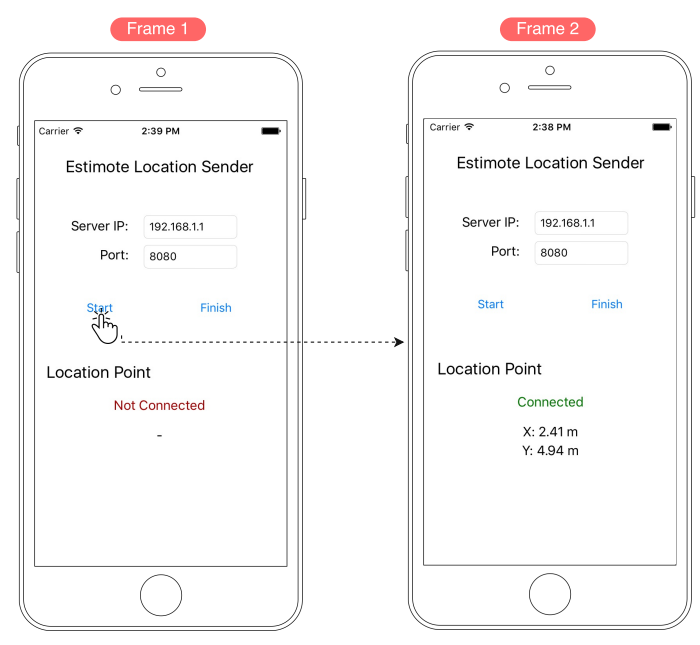

Fig. 4. E-Presence IPS appflow

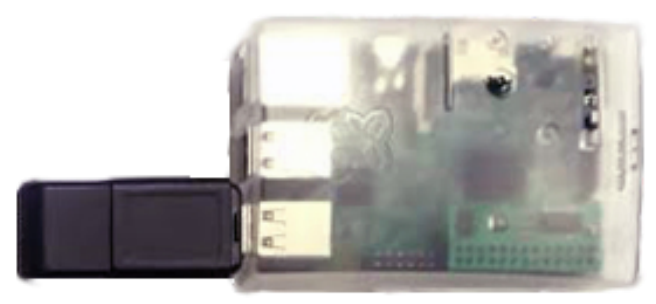

Fig. 5. Card reader setup used to prototype the system

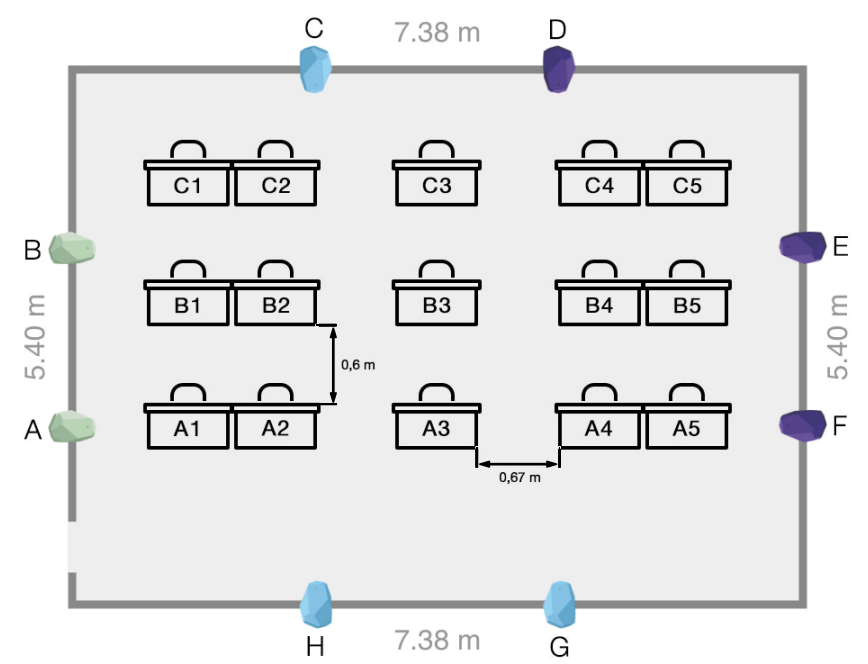

Fig. 6. Scheme of classroom used to evaluate the system. 
an IPS capable of running in an Android environment, the use of the Raspberry card reader will no longer be necessary. A solution using only an iOS device is also possible as the iPhone models 7, 7 Plus, 8, 8 Plus, X, XS, XS Max and XR support MIFARE Tag reading.

\section{Methodology and Evaluation Performance}

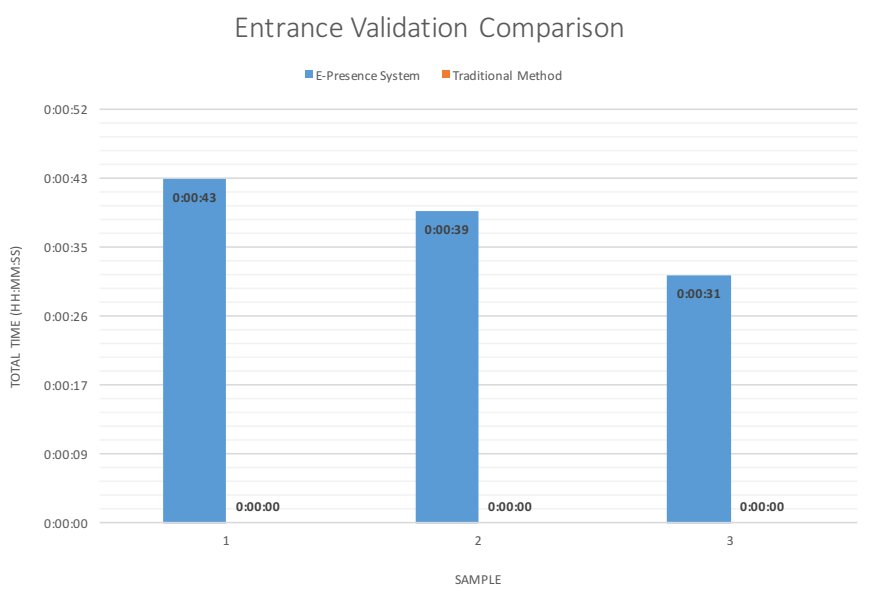

Fig. 7. Entrance validation time comparison results

To perform the system evaluation, a group of 10 students was used during three different assessment activities to verify and validate the actual behaviour against the expected behaviour of the system.

Before starting the evaluation, it was first explained how the system operates in each phase of the evaluation activity. To guarantee the same conditions in the different samplings, each student was asked at the beginning of each activity to have his/her student card available.

After starting the activity, during the Entrance Validation phase, each student validated his student card at the entrance, then read the assigned seat and followed away. During this stage, the Mean Service Rate metric was measured based on the conditions imposed. This time metric was measured from the moment the student followed to validate his card until he/she left for the assigned location.

Later, during the In-Place Validation phase, the faculty member started by validating each row, proceeding to the next one after the previous row validation was completed. Here, the Mean Service Rate metric was measured from the moment the faculty member arrives next to a seat until he left for the next valid place. To compare with the traditional method, a second In-Place validation was made. This time, after drawing the layout map of the classroom, the faculty member registered the information of each student card, asking the student to validate and sign the recorded data.

Finally, during the Delivery Validation, each student delivers the evaluation's answer sheets and confirms their delivery by passing their student card through the NFC sensor. Additionally, the teacher validates the collection of the finalised exam sheets through the application. Then again, the Mean Service
Rate metric was measured from the moment the student follows to validate his student card near the NFC reader until the faculty member validates the collection of the finalised exams through the application. For comparative purposes, the students were asked to deliver the evaluation's answer sheets again, but the validation was only completed as soon as the student signed a delivery evaluation sheet.

D. Results

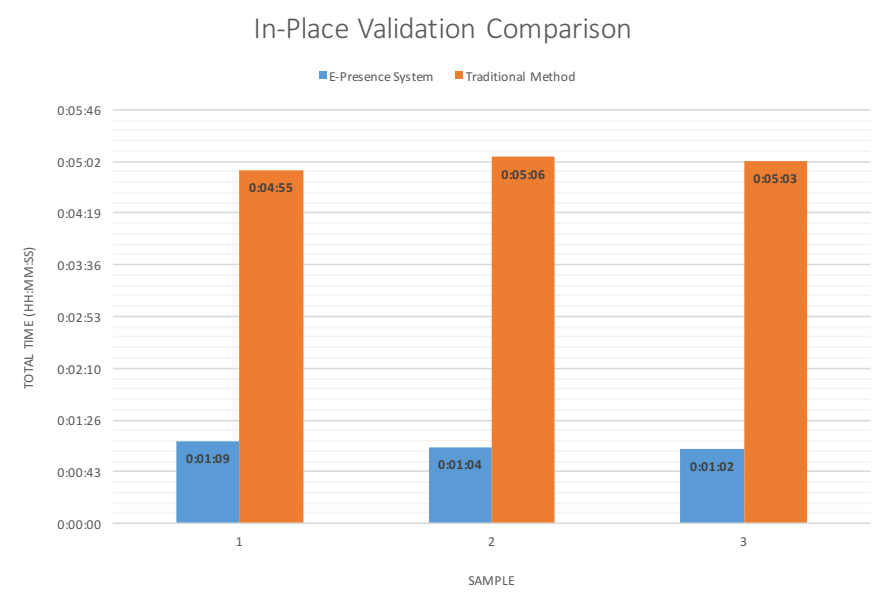

Fig. 8. In-Place validation time comparison results

This section intends to evaluate the performance of the system against the traditional method in each phase of the evaluation activity.

1) Entrance Validation: By analysing the chart in Figure 7 , it can be concluded that there is a disadvantage of the EPresence system when compared to the traditional method during the Entrance Validation phase. This delay occurs because the student has to validate his student card and will have to check the seat location assigned by the system to where it should be headed to.

In contrast to the traditional method, this delay doesn't occur since every student follows directly up into the classroom to a random row and seat position. For this reason, the entrance time was not considered using the traditional method.

However, if the student's enrollment in the evaluation activity is verified on a list of enrolled students, this process will be slower.

2) In-Place Validation: Considering the chart in Figure 8, it can be concluded that the E-Presence system has a much better performance than the traditional method during the InPlace Validation phase.

Unlike a more traditional approach where the teacher has to verify the id and register, or log, the location of the student in the exam room.

The developed system abstracts the interaction between the faculty member and the student, avoiding undesirable interruptions of the student during the exam. This is a timeconsuming and error-prone process, particularly in situations where the evaluation activity environment is crowded. For this 
comparison was also not considered the time to create the map of the classroom for the logging activity, that can be a difficult task to perform in a large classroom.

Another additional point is that the E-Presence system guarantees that the student is in fact on the assigned seat, since it uses the IPS to verify the geographical location at the moment of validation, ensuring fraud control.

3) Delivery Validation: As illustrated by the chart presented in Figure 9, it can be concluded that the E-Presence system also has a better performance over the traditional method during the Delivery Validation phase.

Similarly to the process using the conventional method and analysed in Sub-Section V-D2, the faculty member has to find the students' registration information to validate their delivery. Also, another standard way of proving deliveries is by signing a delivery sheet. However, both methods are slower than using the E-Presence system.

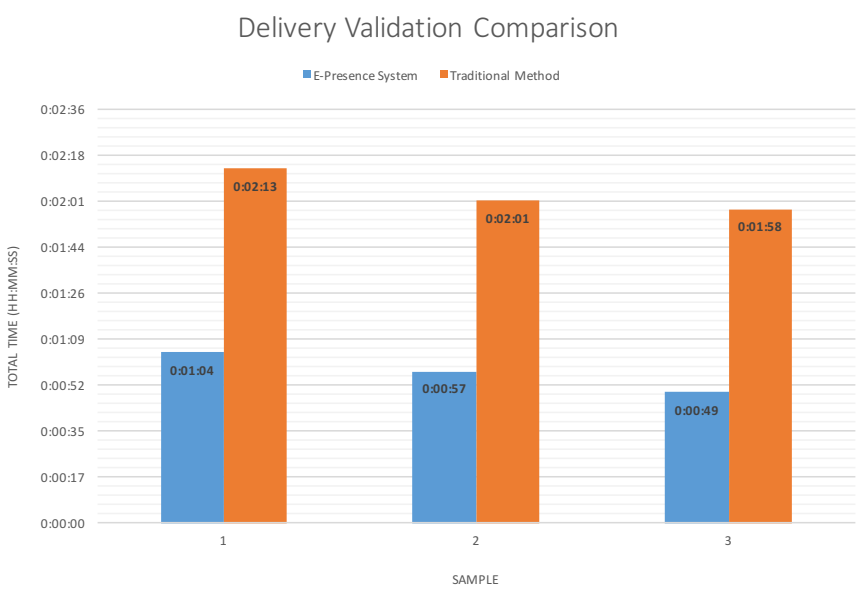

Fig. 9. Delivery validation time comparison results

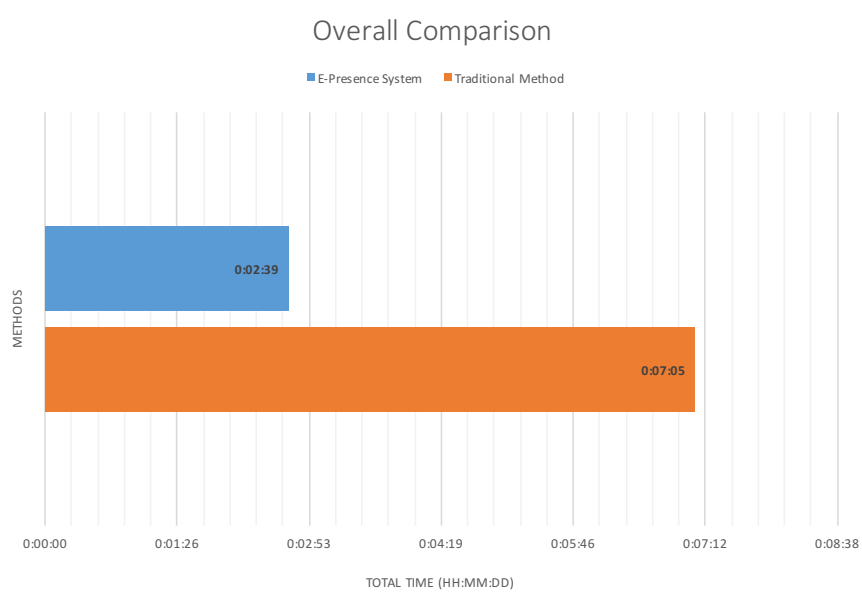

Fig. 10. Total time comparison results

4) System Overview: Although the developed system presents a below performance during the entrance validation phase, the overall performance during the evaluation activities is higher when compared to the traditional control method, as can be analysed in the chart of Figure 10. When compared to the conventional method, the E-Presence system shows a performance $2.7 \mathrm{x}$ higher, which represents $63 \%$ of time performance optimisation.

Moreover, when the evaluation activity is finished, all information obtained during the activity is already in a digital format, leaving the task of uploading all the information in the management platform manually, as it is usually done when following traditional control methods by faculty members. The exported data can be easily configured to a different format, compatible with others faculty's information management platforms.

The usage of the system also allows a greater detail of control over each evaluation activity phases. This kind of information granularity can be useful for solving problems of suspected fraud or to optimise the performance of the assessment activities. As an example, as demonstrated by the chart of Figure 11, it can be seen that In-Place validation is the phase that takes longer to perform when compared to the other phases. To optimise this phase, more than one system could be used at the same time to increase performance.

\section{CONCLUSIONS AND Future WORK}

Departing from a well known problem in the diverse educational systems during the evaluation activities, we proposed, through a mobile and easily adaptable system, a solution capable of helping and assisting lecturers during written exams to prevent academic dishonesty. To test and validate the increase of efficiency by the proposed solution, a prototype was implemented to be tested using a real-case scenario to evaluate the performance of the system against the traditionally used methods.

The evidence collected suggests that the developed system has more benefits than the traditional manual method, which in addition to the performance obtained ensures a rigorous control and fraud prevention in the evaluation activity. In addition to a detailed record about each phase of the evaluation activity, the work performed by the teacher during and after the activity is also reduced through this approach.

As future work, we intend to develop a single multiplatform solution and reduce the cost of the system by using a single mobile device, capable of reading MIFARE/NFC cards and communicate with the IPS without resorting to other external devices. It is also intended to develop the multiserver mode, which allows more than one device to control the same evaluation activity in different classrooms and create a management platform to facilitate the integration and communication of data between the facultys information systems and our application. Other features like path optimisation during the beginning of the activity and control of environmental conditions such as noise and temperature for quality control of the activity environment will be implemented later. 


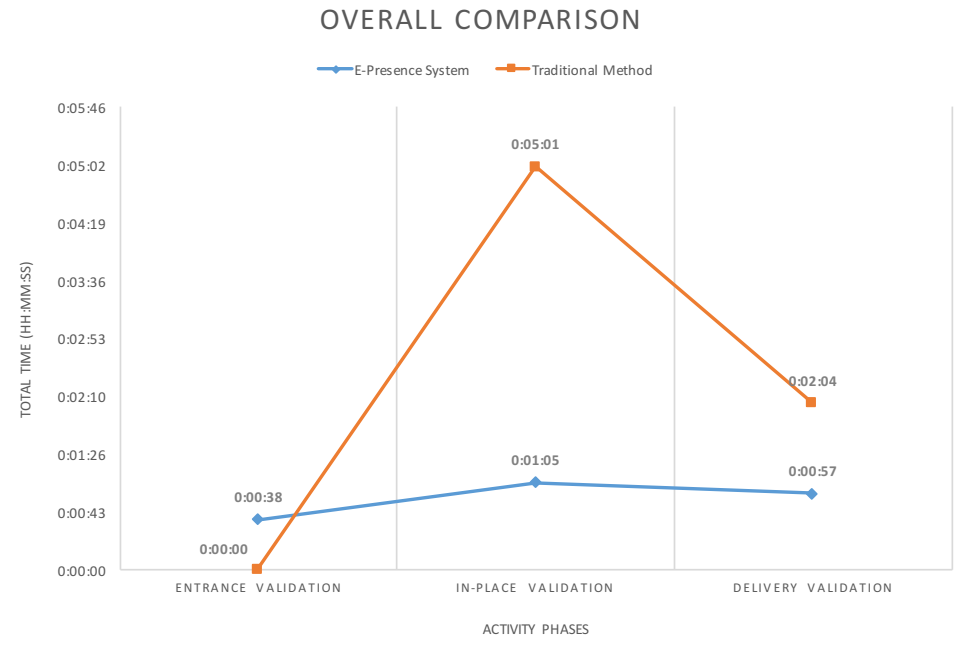

Fig. 11. Overall performance comparison results

\section{ACKNOWLEDGMENT}

We thank NOVA LINCS Automated Software Engineering team and NOVA LINCS Research Laboratory (Ref. UID/CEC/04516/2013) for their financial support.

\section{REFERENCES}

[1] D. EMC. (2016) Education it solutions - education technology emc. Last access: December 2016. [Online]. Available: https://middleeast.emc.com/industry/public-sector/education-it.htm

[2] IBM. (2016) Ibm - education technology solutions. Last access: December 2016. [Online]. Available: https://www935.ibm.com/industries/education/

[3] P. C. Shon, "How college students cheat on in-class examinations: Creativity, strain, and techniques of innovation," Info: Ann Arbor, MI: MPublishing, University of Michigan Library, vol. 1, 2006.

[4] D. W. Bedford, J. R. Gregg, and M. S. Clinton, "Preventing online cheating with technology: A pilot study of remote proctor and an update of its use," Journal of Higher Education Theory and Practice, vol. 11, no. 2, pp. 41-59, 2011.

[5] V. R. Basili, G. Caldiera, and H. D. Rombach, "Goal Question Metric Paradigm," Encyclopedia of Software Engineering, vol. 1, pp. 528-532, 2001.

[6] E. Inc. (2016) Indoor location accuracy problem - Indoor Location - Estimote Community Forums. Last access: November 2016. [Online]. Available: https://forums.estimote.com/t/indoor-locationaccuracy-problem/2710/3

[7] N. Semiconductors. (2016) Mifare classic — nxp. Last access: November 2016. [Online]. Available: http://www.nxp.com/products/identificationand-security/mifare-ics/mifare-classic: $\mathrm{MC}_{4} 1863$

[8] I. Inc. (2016) Scl3711 contactless usb smart card reader [Online]. Available: http://files.identiv.com/products/smart-card-

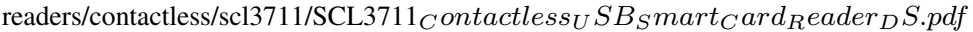

[9] E. Inc. (2016) Estimote beacons - product comparison. Last access: November 2016. [Online]. Available: http://estimote.com/products/ 